\title{
The Graph-DCK Scale: a measure of dorsal column function after spinal cord injury
}

\author{
Marlon L Wong $\mathbb{D}^{1,2} \cdot$ Jacqueline Tibbett $\mathbb{D}^{1} \cdot$ Temitope Adedolapo $^{1} \cdot$ Eva Widerstrom-Noga ${ }^{1}$
}

Received: 4 September 2018 / Revised: 21 November 2018 / Accepted: 10 December 2018 / Published online: 16 January 2019

(c) International Spinal Cord Society 2019

\begin{abstract}
Study design Cohort/psychometric study.

Objectives Assessment of dorsal column medial lemniscus (DCML) function is important for the clinical evaluation of people with spinal cord injury (SCI) because it provides useful information to guide rehabilitation and for prognosticating outcomes. For example, research suggests that damage to the DCML pathway may be associated with neuropathic pain after SCI. Tests for graphesthesia and directional cutaneous kinesthesia (DCK) are commonly used clinically to assess DCML function. However, the reliability and validity of these assessments in people with SCI have not been investigated. Moreover, there is a poor consistency between studies in the methodology of graphesthesia and DCK assessment. The purpose of this study was to determine the psychometric properties of the Graph-DCK Scale, which is a simple and potentially useful scale for assessing graphesthesia and DCK. The Graph-DCK Scale does not require expensive equipment and it takes less than 5 min to administer.
\end{abstract}

Setting A university-based laboratory in Miami, FL, USA.

Methods Sixty-seven people with chronic SCI and neuropathic pain were included in this study. The Graph-DCK Scale and vibration detection were measured twice in each participant, with a two- to four-week period between measurement sessions. Results The scale demonstrated an excellent internal consistency (Cronbach's alpha $>0.90$ ) and test-retest reliability (ICC values $>0.80$ ). Further, convergent validity was supported by moderate and significant positive correlations to vibration detection $(r$ values $>0.40)$.

Conclusion The Graph-DCK Scale is quick and easy to administer, and it provides a reliable and valid assessment of DCML function in people with SCI.

Sponsorship Craig Neilsen Foundation.

\section{Introduction}

For people with spinal cord injury (SCI), the assessment of dorsal column medial lemniscus (DCML) function provides important information regarding sensory status, which may

Supplementary information The online version of this article (https:// doi.org/10.1038/s41393-018-0236-2) contains supplementary material, which is available to authorized users.

Marlon L Wong

mwong2@miami.edu

1 Miami Project to Cure Paralysis, Miller School of Medicine, University of Miami, Miami, Florida, USA

2 Department of Physical Therapy, Miller School of Medicine, University of Miami, Miami, Florida, USA be helpful in guiding its clinical management and for prognosticating the outcomes [1]. Knowledge of DCML function allows for setting reasonable goals in rehabilitation, and DCML function is also predictive of motor outcomes [2] during rehabilitation and neuropathic pain [3-5]. DCML function often improves during the subacute phase after SCI [6], and one study found that 39\% of people classified as having complete SCI (ASIA-A) had some preserved sensory function below the level of injury [7]. Thus, clinically useful tools with adequate test-retest reliability are needed [7].

A variety of methods designed to assess DCML function exist, including light touch detection, vibration detection, two-point discrimination, joint position sense, graphesthesia, and directional cutaneous kinesthesia (DCK). However, these tests often yield different conclusions [8]. For example, in some patients two-point discrimination is impaired 
while vibration detection is intact. In fact, most of these somatosensory modalities involve the complex integration of numerous neuronal inputs, which may contribute to the discrepancy in findings between these tests [8]. Additionally, for most of these somatosensory modalities the reliability and clinical utility are adversely affected by a poor standardization of procedures in clinical practice and research. For example, it is difficult for clinicians to gauge the amount of force exerted when applying the apparatus for two-point discrimination, and the amount of force used impacts the results. As a result, there are disparate reports on normative values for two-point discrimination [9-11]. Similarly, vibration detection is usually assessed clinically using a tuning fork and pressure consistency on applying the tuning fork, or a lack thereof, influences the findings [12].

Quantitative sensory testing research protocols frequently test vibratory sense to evaluate and quantify DCML function $[3,13]$ in a standardized and reliable manner. However, simpler protocols for clinical use are needed. It has been contended that graphesthesia and DCK are the only true tests of DCML function, because these measures rely on the input from rapidly adapting cutaneous mechanoreceptors, and only the axons of rapidly adapting cutaneous mechanoreceptors remain in the DCML for their full extent [8]. Thus, graphesthesia and DCK appear to have less redundancy of input and are likely better measures of DCML function than the other tests of DCML function $[5,8,14,15]$. However, the reliability and validity of methods for assessing graphesthesia and DCK has not been investigated in people with SCI, and there is a poor consistency across studies of people with SCI regarding the methodology of graphesthesia and DCK assessment $[5,8,16,17]$.

DCK, the ability to discern the direction of movement when a stimulus is applied across the skin, and graphesthesia, the ability to recognize letters or numbers written on the skin purely by the sensation of touch, are dependent on inputs from the DCML for adequate performance $[5,8]$. DCK is thought to provide the essential input for graphesthesia, while graphesthesia performance requires additional cerebral processing for the identification of numbers and shapes $[18,19]$. Since graphesthesia places a greater demand on intact patterning of input than does DCK, it is more sensitive to impairment of DCML function than DCK, as demonstrated by Bender et al. [8] in a study of 558 neurologically compromised patients. Tests for DCK and graphesthesia are also quick and easy to perform, and they do not require any expensive equipment. Therefore, DCK and graphesthesia may be ideal methods for the clinical assessment of DCML function after SCI.

The purpose of this study was to evaluate the psychometric properties of a simple scale for assessing graphesthesia and DCK in people with SCI. Current approaches for assessing graphesthesia and DCK typically have dichotomous outcomes (i.e., impaired or normal) or rely on patient self-report (i.e., additional scoring options are provided for the report of altered sensation), which may confound the results. This scale is a performance-based measure with scaled outcome scores. The scale consists of seven different tasks, with a potential score of 0 or 1 for each (representing incorrect or correct performance, respectively), and it provides a potential sum score range of 0-7. This comprehensive and scaled approach to assessing graphesthesia and DCK may be more sensitive to changes in DCML function than the isolated use of tests with dichotomous outcomes.

\section{Methods}

\section{Participants}

This study was based on a subset of data from a larger study involving people with SCI and neuropathic pain [7]. Seventy-two consecutive people with SCI and neuropathic pain were enrolled, and five were eliminated from final data analysis due to incomplete datasets. Participants were at least 18 years old and were able to speak and understand English. Participants were excluded if they had cognitive impairment (a score of $<20$ on the Mini-Mental State Exam), major depression, current (within a year) drug abuse, or significant chronic health conditions other than SCI. The average pain intensity was calculated from the average pain rating (0-10 Numeric Rating Scale) over the past week for up to three pain problems.

\section{Sensory assessments}

Graphesthesia and directional cutaneous kinesthesia testing (Graph-DCK), light touch detection, and vibration detection were assessed in areas of cutaneous innervation at the level of injury and below the level of injury. The level of injury was determined according to the International Standards for Neurological Classification of Spinal Cord Injury guidelines and defined as the most rostral single level with normal sensation [20]. Below level of injury was defined as areas at least four dermatomes below the neurological level of injury. Standardized test sites were identified based on anatomical landmarks to ensure that the same site could be accurately located in each person. Light touch detection was also performed according to the International Standards for Neurological Classification of Spinal Cord Injury guidelines. We certify that all applicable institutional and governmental regulations and approvals concerning the ethical use of human volunteers were followed during the course of this research. 
Table 1 Graph-DCK Scale

\begin{tabular}{lll}
\hline Task & Scale items* & Scoring \\
\hline DCK assessment & Direction (caudal) & $0-1$ \\
Line 1 & Direction (rostral) & $0-1$ \\
Line 2 & Speed (fast or slow) & $0-1$ \\
Graphesthesia assessment & & \\
Number identification & 2 & $0-1$ \\
Number identification & 3 & $0-1$ \\
Shape identification & Square & $0-1$ \\
Shape identification & Circle & $0-1$ \\
Total score range & & $\mathbf{0 - 7}$ \\
\hline
\end{tabular}

$D C K$ directional cutaneous kinesthesia

A score of 0 or 1 was awarded for incorrect or correct identification

* Scale items were administered in a randomized order.

\section{a. Quantitative sensory testing}

Vibration detection measurements were obtained with the TSA-II Neurosensory Analyzer and accompanying software (Medoc Ltd., Ramat Yishai, Israel). The handheld VSA3000 probe of the Medoc system was used to measure vibration detection thresholds for a $100 \mathrm{~Hz}$ stimulus frequency using the ascending method of limits [3]. Participants were instructed to verbally indicate the "first moment" that they felt the vibration sensation, at which time the experimenter stopped the trial and recorded the threshold. This procedure was repeated three times at each test site, and the mean value across the three trials was calculated at the vibration threshold for each site. Vibration thresholds were then transformed into $Z$ scores based on normative data [7].

\section{b. Graphesthesia and directional cutaneous kinesthesia testing}

For assessment of Graph-DCK, the experimenter used the rounded back-end of a pen to lightly trace on the participants' skin, while their eyes were closed. The following tasks were performed in a random order: identification of the 1) direction (caudal or rostral) and 2) speed (fast or slow) of a 4-inch line drawn, 3) identification of the numbers 2 and 3, and 4) identification of the shapes of a circle and square drawn. For each trial, the participants were told if they needed to identify a number or shape, but they were not aware of which order numbers or shapes were being used in the protocol. All procedures were first performed on the cheek to familiarize the participants to the procedures. The slow line was drawn over a total of $4 \mathrm{~s}$. The fast line was drawn over $1 \mathrm{~s}$, and the pen was held on the skin at the terminus for an additional $3 \mathrm{~s}$ so that both the slow and fast trials lasted $4 \mathrm{~s}$. One point was awarded for the identification of the correct direction of each line and an additional point was awarded for the correct identification of the speed of one line relative to the other line, providing a potential total sum score range of $0-3$ for the identification of direction and speed of a line drawn. Similarly, numbers (two trials) and shapes (two trials) were drawn in a random order, over a $4 \times 4$ inch area, and scored as correct or incorrect ( 0 or 1 , respectively) for the identification of the number or shape drawn. The sum score for all the tasks was then calculated (for a potential range of $0-7$, with 7 indicating correct performance on all tasks) and recorded as the Graph-DCK Scale score (Table 1).

\section{Analyses}

\section{Internal consistency}

Cronbach's alpha test was used to analyze the internal consistency between scale items and to determine if all the items should be retained in the scale. A good internal consistency was defined as a Cronbach's alpha score above $0.70[21]$.

\section{Test-retest reliability}

To assess reliability, we administered a scale to 67 participants with chronic SCI and neuropathic pain. Since these participants were in the chronic stage after injury, changes in DCML function were unlikely. The scale was administered twice, with a two- to four-week period between testing sessions, and Intraclass Correlation Coefficient (ICC) estimates and their $95 \%$ confidence intervals were calculated to determine the test-retest reliability of Graph-DCK Scale scores. Good reliability was defined as statistically significant $(p<0.05)$ ICC values above 0.70 based on a singlerater, consistency, 2-way random model [22].

\section{Convergent and divergent validity}

To determine convergent validity, the correlation between mean Graph-DCK Scale scores and normalized vibration detection threshold values, another measure of DCML function, was analyzed. Since the repeated Graph-DCK Scale scores were strongly inter-correlated with initial scores $(r=0.84, p=0.00$ for at level and $r=0.85, p=0.00$ for below level), the mean value of Graph-DCK Scale scores for the two measurement sessions was calculated to provide a more accurate representation of true Graph-DCK Scale performance than a single-session performance. Vibration detection is less specific to DCML function than graphesthesia and DCK [8]. Thus, only a moderate, significant positive correlation between Graph-DCK Scale 
scores and vibration detection was expected. A statistically significant Pearson Product Correlation Coefficient value greater than 0.30 is indicative of a moderate correlation and would be supportive of convergent validity [23]. Divergent validity was determined by analyzing the correlation between mean scale scores and Beck's Depression Inventory scores. Depression is associated with pain after SCI [24], and there was a wide spectrum of Beck's Depression Inventory scores in this cohort (ranging from 0 to 36 ). However, depression is not mediated by DCML function. Thus, the lack of any statistically significant correlation between the scale and the Beck's Depression Inventory would be considered supportive of divergent validity. We also used hierarchical linear regression analysis to assess to control for potential confounders such as age, medication use, and pain in the area tested, since these factors may influence the validity and/or reliability of the scale in people with SCI.

\section{Results}

Sixty-seven participants with SCI and neuropathic pain were included in this study (Table 2). All participants were tested below the level of injury, and 52 were also tested at the level of injury. More than half of the participants had pain in the area being tested, with $63 \%$ reporting pain in the site of testing for at-level Graph-DCK assessment and 55\% reporting pain in the below-level test site. The mean pain intensity was $6.1 \pm 1.8$. Moreover, participants without pain in the at-level test site had significantly better Graph-DCK scores than those with pain in the test site, with a mean difference of $1.6 \pm 0.65(p=0.02)$. The mean Graph-DCK score was $6.3 \pm 1.5$ for participants without pain in the at-level test site and $4.8 \pm 2.5$ for participants with pain in the at-level test site. There was no difference between participants without and those with pain in the below-level test site, with mean scores of $2.2 \pm 2.4$ and $2.6 \pm 2.5$, respectively.

As expected, below-level Graph-DCK scores were significantly higher for participants with an incomplete SCI compared to those with a complete SCI, with mean scores of 4.4 and 1.2, respectively. Additionally, at-level GraphDCK scores were higher than below-level scores for all participants with mean values of $5.0 \pm 2.4$ and $3.5 \pm 2.5$, respectively. Approximately half of the participants demonstrated normal graphesthesia (mean Graph-DCK score of $7, n=29$ ) at the level of injury and approximately half demonstrated near-complete agraphesthesia (mean Graph-DCK score $\leq 1, n=31$ ) below the level of injury. Similarly, $47 \%$ of the participants demonstrated normal light touch sensation at level, and $61 \%$ had absent light touch below level. However, among participants with
Table 2 Participant background information

\begin{tabular}{ll}
\hline & Mean (SD) \\
\hline Age (years) & $38.3(10.9)$ \\
Years with SCI & $11.3(9.1)$ \\
Beck's Depression Inventory (0-63) & $8.8(8.6)$ \\
Numeric Pain Rating Scale (0-10) & $6.1(1.8)$ \\
Pain distribution (\%) & \\
Pain at the level of injury & 63 \\
Pain below the level of injury & 55 \\
Pain both at level and below level & 18 \\
\% With complete impairment (AIS-A) & 58 \\
Cervical SCI (\%) & 52 \\
Thoracic SCI (\%) & 49 \\
Medication use (\%) & \\
Antispasticity & 37 \\
Anti-inflammatory & 27 \\
Anticonvulsant & 25 \\
Opioid & 25 \\
Muscle relaxers or sedatives & 9 \\
Antidepressants or selective serotonin reuptake & 8 \\
inhibitors & \\
Light touch at level (\%) & \\
Absent & \\
Impaired & \\
Normal & 27 \\
Light touch below level (\%) & \\
Absent & \\
Impaired & 15 \\
Normal & \\
\hline & \\
& \\
\hline
\end{tabular}

some but not a total impairment of DCML function, there was a relative even distribution of participants across all levels of impairment (Fig. 1a and b).

\section{Internal consistency}

The Graph-DCK Scale items demonstrated a high internal consistency, with Cronbach's alpha values of 0.91 and 0.92 for at-level and below-level assessments, respectively. Moreover, internal consistency was best with all the seven items included. Thus, the Cronbach's alpha value far exceeded the predetermined standard of 0.70 for a good internal consistency.

\section{Test-retest reliability}

Intraclass Correlation Coefficient values were $0.83(95 \%$ confidence interval $=0.72-0.90)$ and 0.85 (95\% confidence interval $=0.77-0.91)$ for at-level assessment and belowlevel assessment, respectively. Thus, the scale demonstrated good test-retest reliability for use at both sites. 

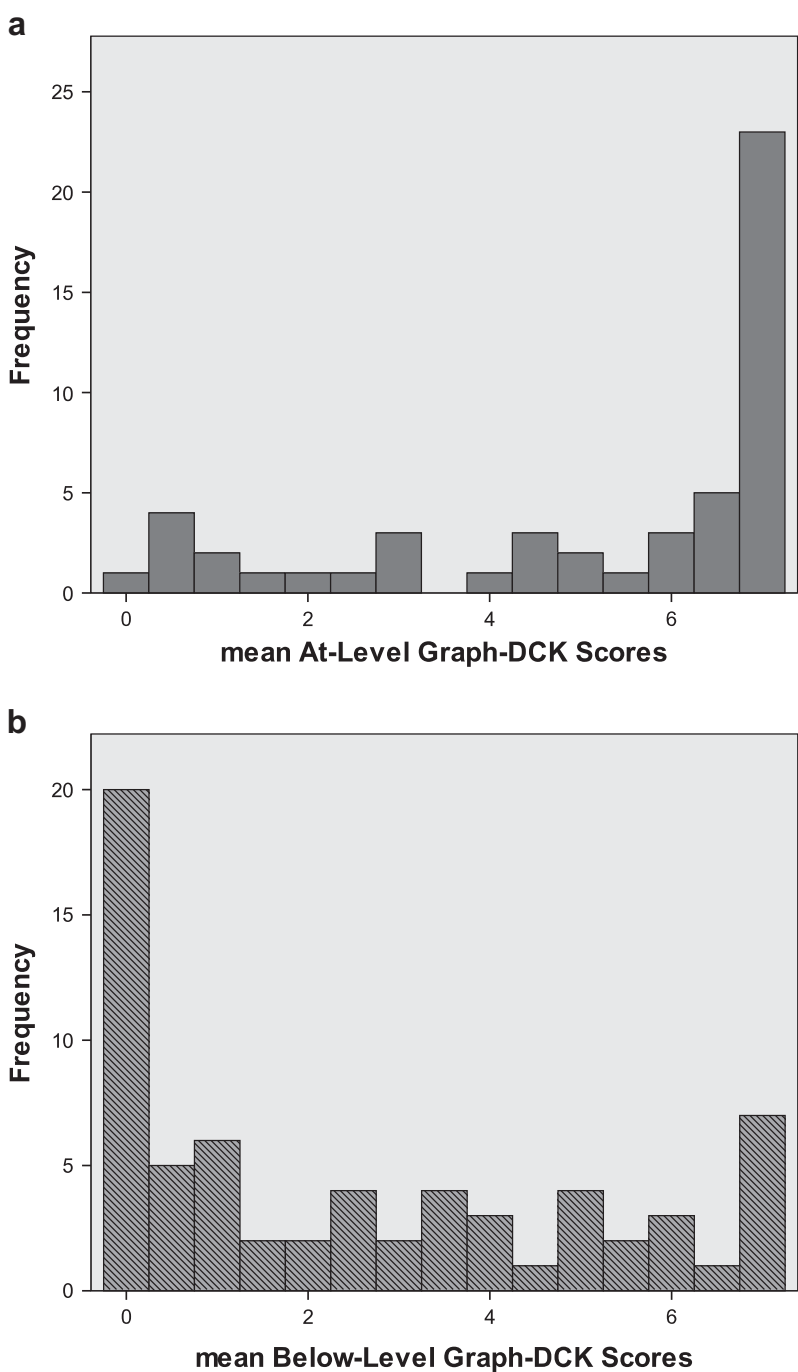

Fig. 1 a and b Distribution of Graph-DCK scores

\section{Validity}

The mean Graph-DCK scores were calculated for each participant from the values taken over the two measurement sessions for at-level and below-level assessments. At-level and below-level mean Graph-DCK scores were significantly correlated to normalized vibration detection scores for both at-level assessment $(r=0.43, p=0.003)$ and below-level assessment ( $r=0.49, p=.000$ ), respectively (Fig. 2a and b). In addition, mean Graph-DCK scores were not significantly associated with Beck Depression Inventory Scores for at-level $(r=0.10, p=0.50)$ or below-level assessment $(r=0.22, p=0.09)$. The moderate correlation observed between Graph-DCK scores and vibration detection provides evidence of concurrent validity for the use of the Graph-DCK Scale as a measure of DCML function, while the lack of association between Graph-DCK scores and depression provides evidence of divergent validity.
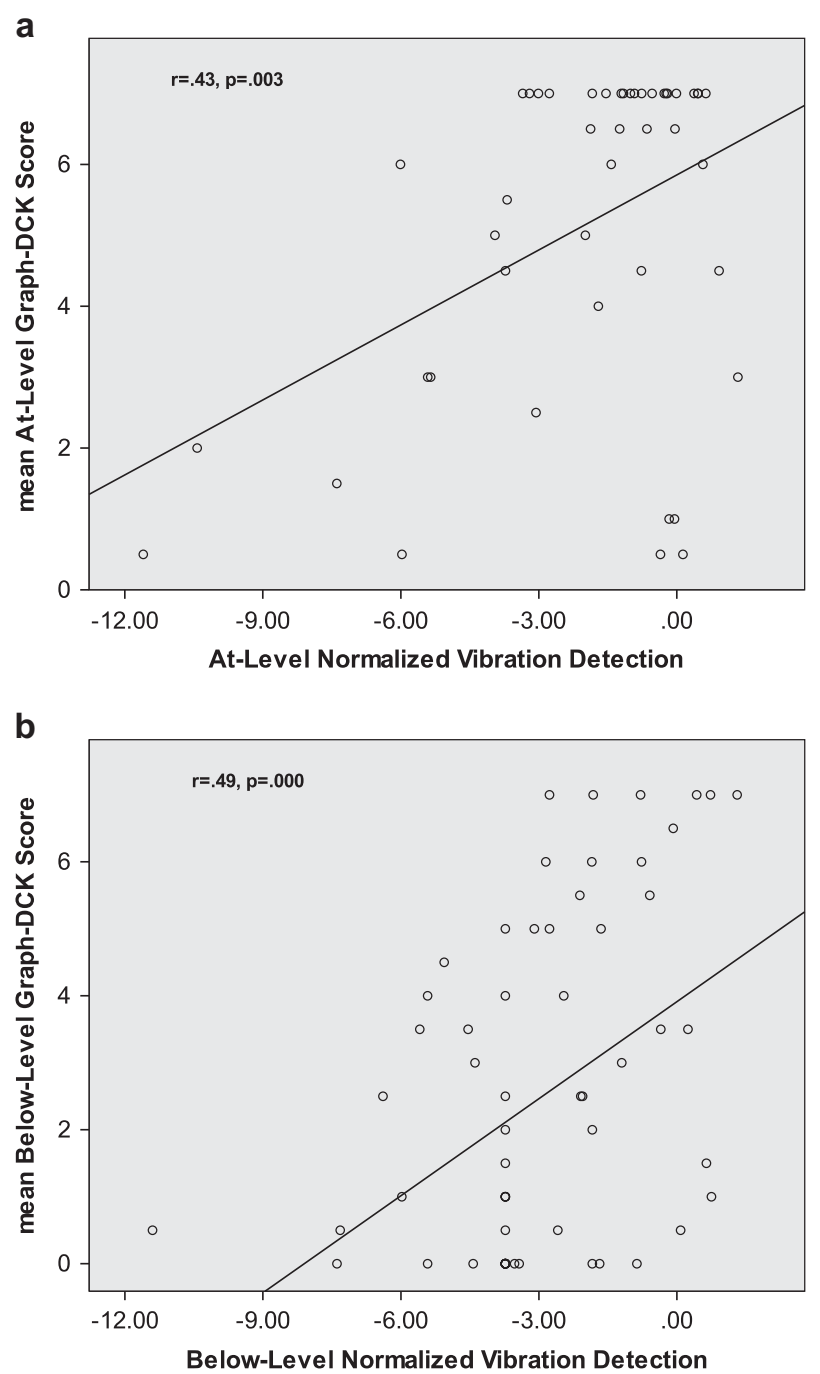

Fig. 2 a and $\mathbf{b}$ The association between Graph-DCK scores and normalized vibration detection

\section{Assessment of potential confounders}

Using hierarchical linear regression analysis, a model including age, medication use (opioid, antispasticity, and anticonvulsant use were entered as separate variables), and pain in the area tested was not predictive of at-level mean Graph-DCK scores $\left(R^{2}=0.07, p=0.68\right)$ or below-level mean Graph-DCK scores $\left(R^{2}=0.11, p=0.19\right)$. Furthermore, partial correlations for the variables entered were not significant within either model, with the exception of age in the below-level Graph-DCK model $(B=0.07, p=0.024)$ (Fig. 3).

\section{Discussion}

The Graph-DCK Scale demonstrated good psychometric properties in a cohort of 67 participants with SCI and 


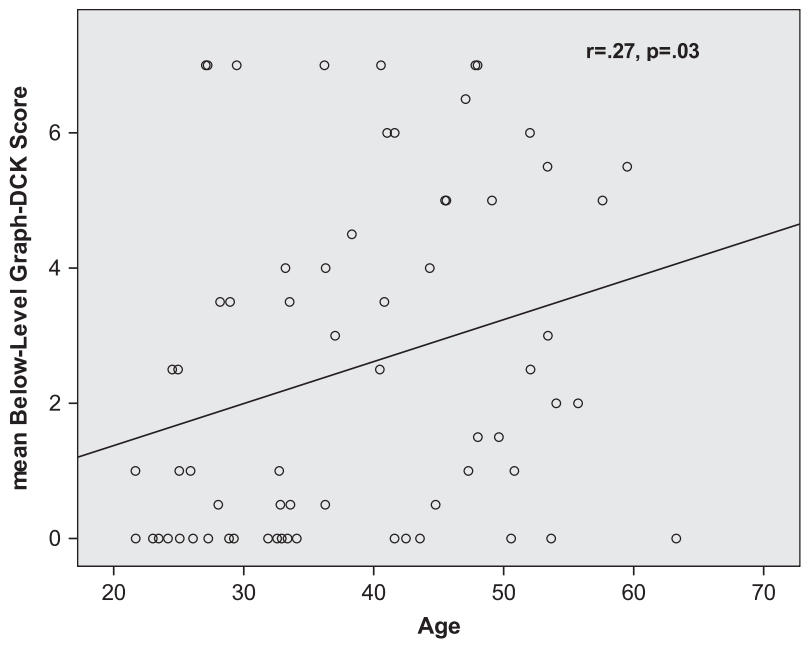

Fig. 3 Scatterplot of mean below-level Graph-DCK scores and age

neuropathic pain, for use at the level of injury and for use below the level of injury. The scale items had an excellent internal consistency (Cronbach's alpha values $>0.90$ ), testretest reliability was good (ICC values $>0.80$ ), and convergent validity was supported by moderate and significant positive correlations to vibration detection ( $r$ values $>0.40$ ). Moreover, performance on the Graph-DCK Scale was not significantly correlated to scores on the Beck's Depression Index, providing evidence of divergent validity, and performance on the scale does not appear to be influenced by medication use. Thus, this scale demonstrates the prerequisite qualities of reliability and validity for its recommended clinical use in people with SCI.

The Graph-DCK Scale described in this study is comprised of items that are all regularly used in clinical practice, and all of the items have been previously described in the literature $[5,16]$. However, previous descriptions of these measures either had a binary interpretation of findings (i.e., impaired or normal) or relied on a subjective feedback to produce scaled scores. Wall and Noordenbos [5] were the first to describe the methods for DCK assessment used in this scale, and they provided a descriptive summary of repeated trials in three different patients. Performances on the correct identification of direction and speed were reported separately, and no attempt was made to quantify the impairment of DCML function by combining findings of graphesthesia and DCK tests. Defrin et al. [16] utilized the exact same items described in this study, and they scored items on a four-point scale $(0=$ complete lack of touch sensation to $3=$ normal touch). However, in the Defrin study [16], scores of 1 and 2 represented reports of abnormal sensation during the test rather than the correctness of performance. Although reports of abnormal sensation are important findings, using the report of abnormal sensation in the scoring introduces several potential confounders that may not be related to DCML function. For example, most people with SCI and some impairment of DCML function are likely to have sensations that would be considered abnormal in the absence of SCI. Therefore, the correct or incorrect dichotomous responses are more precise because of the difficulty in interpreting the report of abnormal sensations.

The goal of the Graph-DCK Scale was to optimize the simplicity and utility of test item interpretation by using a binary scoring system for individual items, while also providing a mechanism to quantify the degree of DCML impairment by summing the scores of binary test items to create a scale. The utility of the Graph-DCK Scale is supported by the strong psychometrics of the scale observed in this study and the broad spectrum of scores observed in this cohort. The high internal consistency between test items suggests that all the test items are capturing the same construct (i.e., DCML function) and supports the validity of summing the item scores to create a scale. The broad spectrum of Graph-DCK scores observed in this cohort suggests that the Graph-DCK Scale captures varying degrees of DCML impairment, which supports the potential clinical utility of this scale.

\section{Limitations and future research}

The findings in this study should be interpreted with a modest degree of caution. This is the first study to investigate the reliability and validity of the Graph-DCK Scale in people with SCI. In fact, we are unaware of any previous studies on the reliability and validity of assessing graphesthesia and DCK in people with SCI. Therefore, these findings need to be replicated. Additionally, the cohort in this study was a relatively homogeneous group of individuals with chronic neuropathic pain after SCI. Future studies are needed to determine the psychometrics of this tool for people in different stages of chronicity after SCI and in those with SCI that do not have neuropathic pain. Further, longitudinal cohort studies are needed to determine the responsiveness of the Graph-DCK Scale to intra-participant changes in DCML function.

\section{Conclusion}

The Graph-DCK Scale provides a reliable and valid method for assessing graphesthesia and DCK in people with SCI. Unlike most methods of quantitative sensory testing or the use of sensory evoked potentials to assess DCML function, this tool can be administered in less than $5 \mathrm{~min}$ and it does not require any expensive equipment. Thus, the GraphDCK scale is a clinically useful method for assessing DCML function in people with SCI. 


\section{Data archiving}

All the data generated or analyzed during this study are included in this published article [and its supplementary information files].

Author contributions MLW was responsible for data analysis and writing the report. JT and TA were responsible for data collection and participated in writing the report. As the senior author, EWN was responsible for designing the protocol, conducting the research, extracting the data, and writing the report.

Funding This study was funded by the Craig Neilsen Foundation.

\section{Compliance with ethical standards}

Conflict of interest The authors declare that they have no conflict of interest.

\section{References}

1. Kirshblum SC, Waring W, Biering-Sorensen F, Burns SP, Johansen M, Schmidt-Read M, et al. Reference for the 2011 revision of the International Standards for Neurological Classification of Spinal Cord Injury. J Spinal Cord Med. 2011;34:54754.

2. Fehlings MG. Essentials of spinal cord injury: basic research to clinical practice. New York, NY: Thieme Medical Publishers; 2013.

3. Cruz-Almeida Y, Felix ER, Martinez-Arizala A, WiderstromNoga EG. Decreased spinothalamic and dorsal column medial lemniscus-mediated function is associated with neuropathic pain after spinal cord injury. J Neurotrauma. 2012;29:270615.

4. Nathan PW, Smith MC, Cook AW. Sensory effects in man of lesions of the posterior columns and of some other afferent pathways. Brain. 1986;109(Pt 5):100341.

5. Wall PD, Noordenbos W. Sensory functions which remain in man after complete transection of dorsal columns. Brain. 1977;100:64153.

6. Reed JL, Liao CC, Qi HX, Kaas JH. Plasticity and recovery after dorsal column spinal cord injury in nonhuman primates. J Exp Neurosci. 2016;10(Suppl 1):1121.

7. Widerstrom-Noga E, Felix ER, Adcock JP, Escalona M, Tibbett J. Multidimensional neuropathic pain phenotypes after spinal cord injury. J Neurotrauma. 2016;33:48292.

8. Bender MB, Stacy C, Cohen J. Agraphesthesia. A disorder of directional cutaneous kinesthesia or a disorientation in cutaneous space. J Neurol Sci. 1982;53:53155.
9. Bell-Krotoski J, Weinstein S, Weinstein C. Testing sensibility, including touch-pressure, two-point discrimination, point localization, and vibration. J Hand Ther. 1993;6:11423.

10. Vriens JP, van der Glas HW. The relationship of facial two-point discrimination to applied force under clinical test conditions. Plast Reconstr Surg. 2002;109:94352.

11. Lundborg G, Rosen B. The two-point discrimination test--time for a re-appraisal? J Hand Surg Br. 2004;29:41822.

12. Rushton EOC, Wright A. C. The assessment of vibration sense in the musculoskeletal examination: Moving towards a valid and reliable quantitative approach to vibration testing in clinical practice. Man Ther. 2011;16:296300.

13. Rolke R, Baron R, Maier C, Tolle TR, Treede RD, Beyer A, et al. Quantitative sensory testing in the German Research Network on Neuropathic Pain (DFNS): standardized protocol and reference values. Pain. 2006;123:23143.

14. Vierck CJ Jr. Alterations of spatio-tactile discrimination after lesions of primate spinal cord. Brain Res. 1973;58:6979.

15. Azulay A, Schwartz AS. The role of the dorsal funiculus of the primate in tactile discrimination. Exp Neurol. 1975;46: 31532.

16. Defrin R, Ohry A, Blumen N, Urca G. Characterization of chronic pain and somatosensory function in spinal cord injury subjects. Pain. 2001;89:25363.

17. Zeilig G, Enosh S, Rubin-Asher D, Lehr B, Defrin R. The nature and course of sensory changes following spinal cord injury: predictive properties and implications on the mechanism of central pain. Brain. 2012;135(Pt 2):41830.

18. Arnold G, Spence C, Auvray M. A unity of the self or a multiplicity of locations? How the graphesthesia task sheds light on the role of spatial perspectives in bodily self-consciousness. Conscious Cogn. 2017;56:10014.

19. Drago V, Foster PS, Edward D, Wargovich B, Heilman KM. Graphesthesia: a test of graphemic movement representations or tactile imagery? J Int Neuropsychol Soc. 2010;16:1903.

20. Kirshblum SC, Burns SP, Biering-Sorensen F, Donovan W, Graves DE, Jha A, et al. International standards for neurological classification of spinal cord injury (revised 2011). J Spinal Cord Med. 2011;34:53546.

21. G SDaN. Health Measurement Scales: a practical guide to their development and use. Fourth ed. Oxford New York: Oxford Univeristy Press; 2008.

22. Koo TK, Li MY. A guideline of selecting and reporting intraclass correlation coefficients for reliability research. J Chiropr Med. 2016;15:15563.

23. Statistical Power JC. Analysis for the behavioral sciences. Hillsdale, NJ: Lawrence Erlbaum; 1988.

24. Ataoglu E, Tiftik T, Kara M, Tunc H, Ersoz M, Akkus S. Effects of chronic pain on quality of life and depression in patients with spinal cord injury. Spinal Cord. 2013;51:236. 